\title{
A Pheromone Based Model for Ant Based Clustering
}

\author{
Saroj Bala \\ AKG Engineering College, \\ Ghaziabad, U.P., India.
}

\author{
S. I. Ahson \\ Shobhit University, \\ Meerut, U.P., India.
}

\author{
R. P. Agarwal \\ Shobhit University, \\ Meerut, U.P., India
}

\begin{abstract}
Swarm intelligence is a collective effort of simple agents working locally but resulting in wonderful patterns. Labor division, decentralized control, stigmergy and self organization are the major components of swarm intelligence. Ant based clustering is inspired by brood sorting in ant colonies, an example of decentralized and self organized work. Stigmergy in ant colonies is via a chemical pheromone. This paper proposes a pheromone based ant clustering algorithm. In the proposed method, ant agents use pheromone to communicate the place to make the clusters, not the path as in real ants. The pheromone concentration helps to decide pick and drop of objects with less parameters and calculations. The simulations have shown good and dense cluster results.
\end{abstract}

Keywords-Ant colony optimization; Clustering; Stigmergy;
Pheromone.

\section{INTRODUCTION}

Swarm intelligence is an area of artificial intelligence based on the working of nature swarms like ants, honey bees, bird flocks, fish schools etc. These swarms are made up of simple agents interacting locally but emerging global patterns. An agent is a subsystem that interacts with its environment consisting of other agents but acts relatively independently from all other agents. The agent does not follow commands from a leader [11]. Ant colony optimization is the most popular swarm. It has been applied to vast variety of optimization problem. It is inspired by collective behavior of ants that are doing complex tasks without any leader. In the early 1990s, ant colony optimization was introduced by $M$. Dorigo and colleagues for the solution of hard combinatorial optimization problems [1]. The inspiring source of ACO is the foraging behavior of real ants.

When searching for food, ants initially explore the area surrounding their nest in a random manner. As soon as an ant finds a food source, it evaluates the quantity and the quality of the food and carries some of it back to the nest. During the return trip, the ant deposits a chemical pheromone trail on the ground. The quantity of pheromone deposited, which may depend on the quantity and quality of the food, will guide other ants to the food source. Indirect communication between the ants via pheromone trails enables them to find shortest paths between their nest and food sources.

Ant based clustering is inspired by brood sorting in ant colonies. Brood sorting shows the decentralized and self organized behavior of ants. Our work is based on combination of this behavior and the stigmergy via the pheromone. We propose here a new ant based clustering method that uses pheromone to mark the places where clusters will be formed.

\section{BASIC Ant Clustering Method}

Firstly Deneubourget. al proposed a basic ant clustering algorithm [2]. He focused on clustering objects by using a group of real-world robots. In his model, the ants would walk randomly on the workspace, picking or dropping one data element from it. The ants possessed only local perceptual capabilities. They could sense the surrounding objects were similar or not to the object, they were carrying. Based on this information, they would perform the pick or drop action. The less objects of the same sort there are in the immediate environment, the greater the probabilities that it will pick it up, as given by the following function:

$$
P_{\text {pick }}=\left(\frac{K^{+}}{K^{+}+f}\right)^{2} \text { where } f \text { is an estimation of the }
$$

fraction of nearby points occupied by objects of the same type, and $\mathrm{K}^{+}$is a constant. The probability thus decreases with $f$, from 1 (when $f=0$ ), to $1 / 4$ (when $f=\mathrm{K}^{+}$), and less as $f$ tends to 1 . The more objects of the same sort there are in the immediate environment, the greater the probability that it will do so, as given by the following function:

$$
P_{\text {drop }}=\left(\frac{f}{K^{-}+f}\right)^{2}
$$

where $f$ is as same and $\mathrm{K}^{-}$is a constant. The probability thus increases with $f$, from 0 (when $\mathrm{f}=0$ ) to $1 / 4\left(\right.$ when $f=\mathrm{K}^{-}$), and more as $\mathrm{f}$ tends to 1 .

This method was further extended by Lumer and Faieta [3] and Gutowitz [4].

\section{PHEROMONE BASED METHODS IN LITERATURE}

Stigmergy, the indirect communication, between the ants via pheromone trails enables them to find shortest path between their nest and food sources. In ant based clustering, many concepts are proposed for pheromone laying. Ngenkaew proposed multiple pheromones [8] in ant based clustering. Trailing pheromone is used to lead ants to return to the nest and foraging pheromone to find the new food source.

Zhang [10] proposed a method which decides the ant movement by using pheromone. It uses pheromone intensity to decide the moving orientation instead of using memory. The ant's transition probability from one place and orientation $\left(a_{1}\right.$, $\left.b_{1}\right)$ to the next place and orientation $\left(a_{2}, b_{2}\right)$ depends on pheromone concentration and current orientation. Before moving to the next grid, the ant will first calculate the pheromone concentration of the eight grids in the surrounding 
environment, if the pheromone concentration of the eight grids is the same, the ant will move towards the original orientation. Due to the grid space of high pheromone concentration, the random moving of ants is avoided that results in acceleration of the convergence of the algorithm. The state of an individual ant has been expressed by a phase variable containing its position $r$ and orientation $\theta$.

Ghosh [6] proposed a method of aggregation pheromone. The ants move with an aim to create homogenous groups. The amount of movement of an ant towards a point is governed by the intensity of aggregation pheromone deposited by all other ants at that point. This gradual movement of ants in due course of time results in formation of groups or clusters. The amount of pheromone deposited by an ant at a particular location depends on its distance from it. Less is the distance; more is the deposition of pheromone. Thus aggregation pheromone density at a particular location depends on the number of ants in its closer proximity. More is the number of ants in its closer proximity; the higher is the aggregation pheromone density. The ants are allowed to move in the search space to find out the points with higher pheromone density. The movement of an ant is governed by the amount of pheromone deposited at different points of the search space. More the deposited pheromone more is the aggregation of ants. This leads to the formation of homogenous groups or clusters. The number of clusters so formed might be more than the desired number of clusters. So as to obtain the desired number of clusters, in the second step agglomerative average linkage algorithm is applied.

Liu [7] proposed an incremental method where each agent computes the information entropy or pheromone concentration of the area surrounding it, and clusters objects by picking up, dropping and moving.

Ramos [9] modified the ant-based clustering by changing the movement paradigm. His ants would move according to a trail of pheromones left on clustering formations. This would reduce the exploration of empty areas, where the pheromone would eventually evaporate. This algorithm was called ACLUSTER.

Chircop [5] proposed Multiple Pheromone Algorithm for Cluster Analysis. Ants detect individual features of objects in space and deposit pheromone traces that guide towards these features. Each ant starts off by looking for a particular feature but they can combine with ants looking for other features if the match of their paths is above a given threshold. This enables ants to detect and deposit pheromone corresponding to feature combinations and provides the colony with more powerful cluster analysis and classification tools.

\section{PROPOSED METHOD}

The existing methods for ant based clustering are mainly based on memory. Whatever an ant finds in its workspace it stores in memory and the pick/drop decisions are based on the probability calculated from the memory. Few methods have used pheromone concept from real ants to change the random walk into biased walk according to pheromone.

The proposed method takes into consideration the random walk for movement and pheromone concept to avoid the memory. According to this the ants will move randomly in the workspace, a 2-D grid, but the pick and drop actions will depend upon the pheromone on a particular cell. Pheromone on a particular cell will depend upon the objects on that particular cell and the objects lying on its neighbor cells. Here, we consider total 9 cells, a cell and its 8-neighbor cells.

The pheromone on a particular cell will be incremented by a fixed amount for every similar object in the surrounding and decremented by the same amount for every dissimilar object. Empty cells will not contribute to the pheromone and all the empty cells will not have any pheromone. Pick/drop actions will be done according to a threshold that changes its value with the cluster construction. An ant, if encounters an object on a cell, will pick it up if the pheromone on that cell is less than the threshold. If a loaded ant encounters an object, same as its load, it drops the object in neighborhood if the pheromone on the cell is greater than or equal to the threshold. The clustering process is presented below. It consists of three steps:

\section{A. Initial pheromone laying:}

This is initialization step. Every location $(i, j)$ with an object on the grid will be assigned a pheromone $\tau_{\mathrm{ij}}$ based on the surrounding. Let $\Delta \tau$ be the amount of pheromone change. The presence of similar objects in the surroundings increases the pheromone trail on the location by $\Delta \tau$ and a dissimilar object decreases the trail by $\Delta \tau$.

\section{B. Cluster construction:}

Ants move randomly on the grid. If an unloaded ant meets an object and finds pheromone on that location below the threshold value, it picks it up. If loaded ant comes to a location with pheromone value greater than the threshold and its load matches with the object on that location, it drops in neighborhood of location with $\mathrm{P}_{\mathrm{drop}}=2 \tau_{i j}$ probability.

\section{Pheromone updation:}

On a pick/drop action, the pheromone on that location and the surrounding location will be updated. On Pickup, $\tau_{i j}=0$ and pheromone in the surrounding cells containing the similar object will be decreased and containing dissimilar objects will be increased. On Drop, $\tau_{i j}=\Delta \tau$ and pheromone in the surrounding cells containing the similar object will be increased and containing dissimilar objects will be decreased.

Steps B and C will alternate until final clusters form.

The number of clusters formed will be influenced by the initial pheromone assigned to a cell which in turn is dependent on the random distribution of data on the 2-D grid. The random distribution on adjacent cells on two different locations may result in two clusters having the similar elements. To merge these clusters, we propose a concept similar to agglomerative ants [12]. Each cluster will be represented by an ant. The ants will merge with the ants carrying clusters of similar elements resulting in the final clusters.

\section{EXPERIMENTAL RESULTS}

The proposed method has been implemented in MATLAB. Different studies have been carried out with different number of objects and ants on 2-D grid. One experiment is done on $30 \times 30$ grid. We took 100 objects of two different types, 50 objects of ' $O$ ' type and 50 objects of ' $\square$ 'type. Number of ants 
taken is 20 . The random distribution of objects and clustering results after 3000 iterations are shown in Fig. 1. The other experiment is shown in Fig. 2.

Here, we have taken 200 objects on $40 \times 40$ grid with 40 ants. All the objects are of " $\circ$ ' type, half in blue and half in red color. In the simulations, the initial threshold is taken as 0.1 . Later the threshold value has been updated by $\Delta \tau(=0.01$ here $)$ with cluster construction steps.
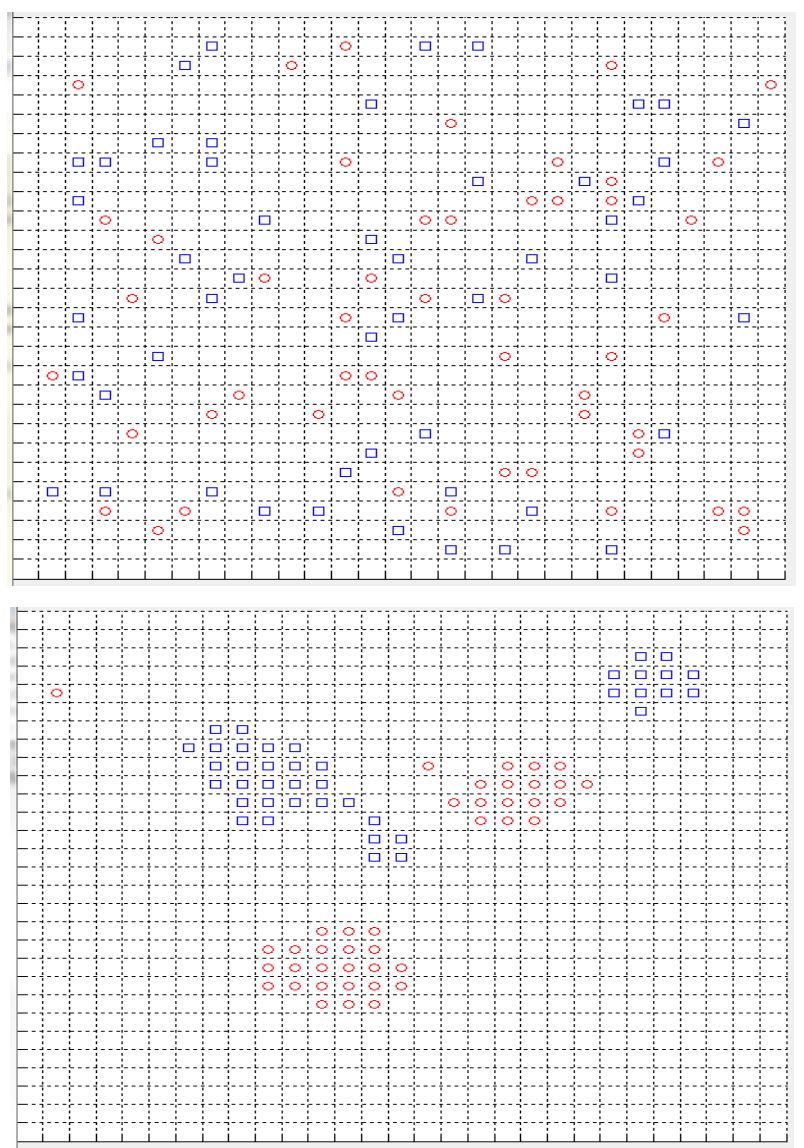

Fig. 1: Random distribution of 100 objects on $30 \times 30$ grid and clusters after 3000 iterations

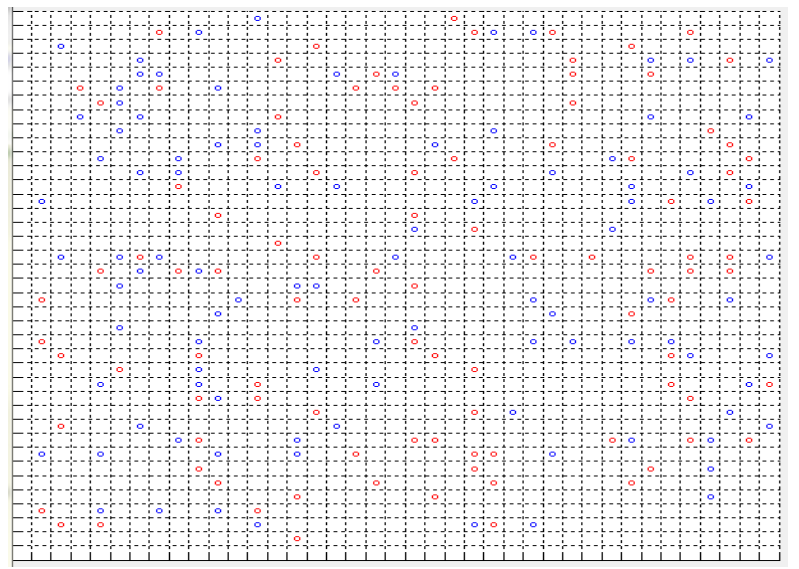

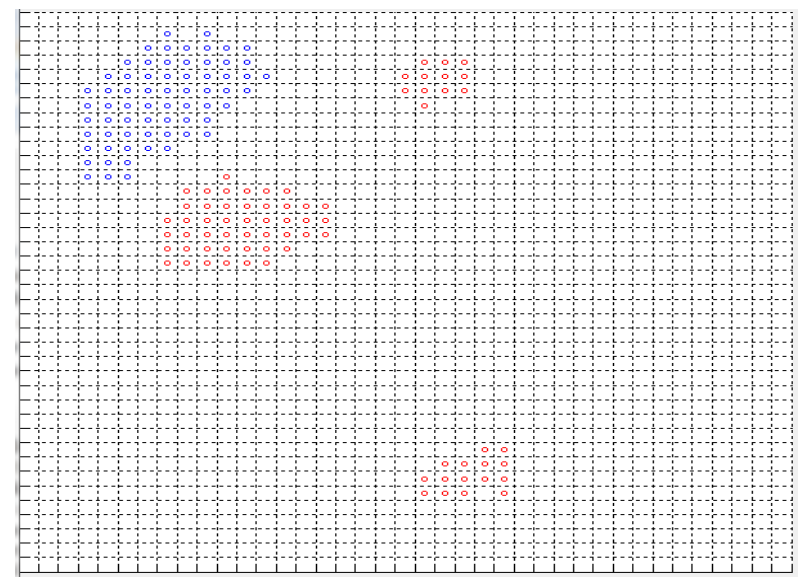

Fig. 2: Random distribution of 200 objects on $40 \times 40$ grid and clusters after 4000 iterations

\section{CONCLUSION}

A new pheromone based method has been proposed for ant based clustering. Locations are carrying pheromones depending on the density of similar objects in the surrounding. The concentration of pheromone helps ant agents to pick and drop objects from a location. The simulations have shown very good and dense clusters. The ants now don't have any memory and need not to store the information of all the objects recently met during its walk. The proposed method needs very less parameters and less calculation. The pheromone will be stored only on the locations equal to number of objects and will be manipulated only on pick and drop actions while in memory based actions, ants update their memory continuously and then calculate the probability accordingly.

\section{REFERENCES}

[1] M. Dorigo, V. Maniezzo and A. Colorni, "Ant system: optimization by a colony of cooperating agents", IEEE Transactions on Systems, Man, and Cybernetics-Part B, Vol. 26, No.1, pp. 1-13, 1996.

[2] J. L. Deneubourg, S. Gross, N. R. Franks, Sendova-Franks, C. Detrain and L. Chretien, "The dynamics of collective sorting: robot-like ants and antlike robots", Simulation of Adaptative Behavior: From Animals to Animats, pp. 356-363, 1991.

[3] E. D. Lumer and B. Faieta, "Diversity and adaptation in populations of clustering ants", Proc. of the Third International Conference on The Simulation of Adaptative Behavior: From Animals to Animats 3, pp. 449508. MIT Press, 1994.

[4] H. Gutowitz, "Complexity-seeking ants", In Proc. of the Third European Conference on Artificial Life, 1993.

[5] J. Chircop, J. and C. D. Buckingham, "A multiple pheromone algorithm for cluster analysis", International conference on swarm intelligence, ICSI 2011, Cergy, France, June 14-15.

[6] A. Ghosh, A. Halder, M. Kothari and S. Ghosh, "Aggregation pheromone density based data clustering", Information Sciences 178, pp. 2816-2831. 2008.

[7] B. Liu, J. Pan and B. Mckay, "Incremental clustering based on swarm intelligence", Proceedings of Simulated Evolution and Learning 6th International Conference (SEAL 2006), Springer Lecture Note in Computer Science 4247 Springer-Verlag April 2006, pp. 189-196.

[8] W. Ngenkaew, S. Ono and S. Nakayama, "Pheromone-based concept in ant clustering", $3^{\text {rd }}$ international conference on Intelligent System and Knowledge Engineering, ISKE 2008, IEEE, pp. 308-312. 
[9] V. Ramos, P. Pina, and F. Muge, "Self-organized data and image retrieval as a consequence of inter-dynamic synergistic relationships in artificial ant colonies", in Frontiers in Artificial Intelligence and Applications, Soft Computing Systems - Design, Management and Applications, IOS Press, Vol. 87, ISBN 1586032976, Santiago, Chile, pp. 500-509, 2002.

[10] F. Zhang,Y. Ma, N. Hou and H. Liu, "An ant-based fast text clustering approach using pheromone", Fifth International Conference on Fuzzy Systems and Knowledge Discovery, FSKD 2008, IEEE, pp. 385-389.
[11] E. Bonabeau, M. Dorigo and G. Theraulaz, "Swarm intelligence: from natural to artificial systems", Oxford university press, 1999 .

[12] S. Bala, S. I. Ahson and R. P. Agarwal, "Agglomerative ants for data clustering", International journal of computer applications, Vol. 47, No. 21, June edition, 2012. 